\title{
Le Moyen Âge vu d'ailleurs IV/La Edad Media desde otros horizontes IV. Sources et concepts/ Fuentes y conceptos
}

Buenos Aires, Academia Nacional de Bellas Artes, $1^{\mathrm{er}}-4$ novembre 2006

\section{Marta Madero et Eliana Magnani}

\section{(2) OpenEdition \\ Journals}

Édition électronique

URL : https://journals.openedition.org/cem/490

DOI : $10.4000 /$ cem. 490

ISSN : 1954-3093

Éditeur

Centre d'études médiévales Saint-Germain d'Auxerre

Édition imprimée

Date de publication : 15 août 2006

ISSN : 1623-5770

\section{Référence électronique}

Marta Madero et Eliana Magnani, «Le Moyen Âge vu d'ailleurs IV/La Edad Media desde otros horizontes IV. Sources et concepts/ Fuentes y conceptos », Bulletin du centre d'études médiévales d'Auxerre / BUCEMA [En ligne], 10 | 2006, mis en ligne le 08 septembre 2006, consulté le 22 septembre 2022. URL : http://journals.openedition.org/cem/490 ; DOI : https://doi.org/10.4000/cem.490

Ce document a été généré automatiquement le 22 septembre 2022.

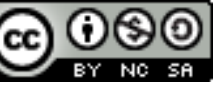

Creative Commons - Attribution - Pas d'Utilisation Commerciale - Partage dans les Mêmes Conditions 4.0 International - CC BY-NC-SA 4.0

https://creativecommons.org/licenses/by-nc-sa/4.0/ 


\section{Le Moyen Âge vu d'ailleurs IV/La Edad Media desde otros horizontes IV. Sources et concepts/ Fuentes y conceptos}

Buenos Aires, Academia Nacional de Bellas Artes, $1^{\text {er }}-4$ novembre 2006

Marta Madero et Eliana Magnani

1 Cette rencontre, réunissant des médiévistes - historiens, historiens de l'art et archéologues - européens et latino-américains, est le prolongement et l'élargissement de trois réunions réalisées à Auxerre en 2002, à São Paulo (Brésil) en 2003 et à Madrid en 2005, à l'initiative du Centre d'études médiévales d'Auxerre (UMR 5594) et soutenues par plusieurs partenaires ${ }^{1}$.

2 Né du constat de la méconnaissance en Europe de la production médiévistique en Amérique latine, pourtant fortement influencée par les écoles historiques françaises et ibériques, ce programme vise à combler cette lacune en diffusant et discutant les recherches en cours sur les deux continents. Par le moyen de rencontres organisées d'après trois grands axes directeurs - l'état de l'historiographie depuis l'après-guerre, l'histoire et l'anthropologie, l'histoire sociale et économique - ainsi que d'une large ouverture aux questions liées à l'archéologie et à l'histoire de l'art médiévales, il cherche à promouvoir et multiplier les contacts entre médiévistes qui ont rarement l'occasion de se rencontrer, stimulant ainsi la création d'un vaste réseau d'échanges scientifiques et humains, dont on observe déjà le développement. Il privilégie la participation des jeunes médiévistes - doctorants et post-doctorants - tout en sollicitant la collaboration des chercheurs et enseignants-chercheurs confirmés.

3 Pour le colloque conclusif du programme, prévu à Buenos Aires en novembre 2006, la réflexion s'articulera autour des sources et des concepts en jeu dans les études de la société médiévale. Peu enclins à la conceptualisation de leurs disciplines, historiens, historiens de l'art et spécialistes de la littérature médiévale, discuteront alors, dans une perspective historiographique et programmatique, les notions mises en œuvre dans 
leurs travaux. Certains concepts utilisés comme allant de soi renvoient souvent au sens commun de notre société contemporaine, sans rendre compte des spécificités de la société médiévale. La recherche des notions propres à rendre au Moyen Age une véritable compréhension restent en grande partie à dégager. Cela passe par le questionnement des sources dans leur diversité - l'écrit, les images, les objets -, leur traitement et les moyens nouveaux de les aborder. Ce sont donc les pratiques historiennes, et celles de ses disciplines sœurs, qui seront au centre des préoccupations.

- Historiographie : approches et bilans (coord. Marta MADERO)

- Histoire médiévale au Chili, Luis ROJAS DONAT

- Anthropologie historique, un bilan, Jean-Claude schмiтT

- Dialoguer entre disciplines est-il possible? Anthropologie, sociologie et histoire médiévale autour du "don", Eliana MAGNANI

\section{$6 \quad 14 h 30-18 h 30$}

\section{Autour de l'Ecclesia (coord. José RIVAIR MACEDO)}

- Hiérarchie : organisation ecclésiale et distinction sociale, Dominique IOGNA-PRAT

- Destins de l'hérésie des Pères latins aux Carolingiens, Alfonso HERNÁNDEZ

- Hérésies et « système d'Église » en Occident (xI -

- 0 « sujeito político » segundo a história da penitência na época gregoriana, Néri DE BARROS ALMEIDA

- Plenitudo potestatis y procesos inquisitoriales en el Concilio de Constanza, Sebastian PROVVIDENTE

7 jeudi 2 novembre, $10 \mathrm{~h}-13 \mathrm{~h}$

Dynamique sociale et politique : seigneurs, communautés et État (coord. Maria Inéz CARZOLIO)

- Os « Reinos Bárbaros » : Estados Segmentários na Alta Idade Média Ocidental, Mario Jorge DA MOTTA BASTOS

- La aristocracia en la España Medieval, Pascual MARTínez sopena

- Las comunidades locales en la España Medieval, Ana RODRIGUEZ LóPEZ

L'écrit (coord. Sylvia DELPY)

- De l'écriture à l'archive: le problème de la source, Joseph MORSEL

- Archéologie des textes médiévaux : méthodes et enjeux pour l'historien, Pierre CHASTANG

- Reflexões teóricas e metodológicas acerca dos manuscritos medievais do "De re coquinaria" de Apicius para a história da alimentação na Alta Idade Média, Wanessa C. ASFORA

- Representar, instituir, redimir : oralidad y escritura en los textos forales, Paola MICELI

\section{vendredi 3 novembre, $10 \mathrm{~h}-13 \mathrm{~h}$}

\section{Le texte historiographique et littéraire (coord. Flavio DE CAMPOS)}

- Trois chroniques pour un roi. L'historiographie du règne de Ferdinand III (1217-1252), Georges MARTIN

- Elementos para una poética del relato cronístico, Leonardo FÚNES 
- Fremosos cantos : reflexões metodológicas sobre a lírica galego-portuguesa, Lênia Márcia MONGELLI

Le texte hagiographique (coord. Ariel GUIANCE)

- « Signum vel res »? La ponderación del milagro en la sociedad visigoda (589- 711), Eleonora DELL' ELICINE

- Cristo, Lázaro y los pobres. La representación de la pobreza en la Castilla medieval, Marcos RUBIOLO GALÍNDEZ

- Los milagros en la religiosidad hispánica (siglos XII al XVI), Gerardo RODRíGUEZ

11 samedi 4 novembre, 9 h30 - 13h

Les objets (coord. Hilário FRANCO JR.)

- Décrire, dire et penser les objets en Histoire de l'art médiéval, Daniel RUSso

- Uma análise serial de relicários antropomorfos medievais, Vivian COUTINHO DE ALMEIDA

- L'apport de trente ans d'études des archives du sol en France : points de vue du médiéviste historien des textes, Monique BOURIN

\section{Conclusion - Marta MADERO}

\section{Participants :}

- Wanessa C. ASFORA (Univ. de São Paulo, Brésil)

- Néri De baRRos ALMEIDA (Univ. de Campinas, Brésil)

- Mario BASTOS (Univ. Federal Fluminense, Brésil)

- Monique BouRin (Univ. de Paris I)

- Flávio DE CAmpos (Univ. de São Paulo, Brésil)

- Maria Inés Carzolio (Univ. Nac. de Rosario)

- Pierre CHASTANG (Univ. de Saint.-Quentin en Yvelines)

- Vivian Coutinho de ALmeIda (Univ. de São Paulo, Brésil)

-Eleonora Dell'elicine (Univ. de Buenos Aires, Univ. Nac. de General Sarmiento)

- Sylvia DELPY (Univ. de Buenos Aires / CONICET)

- Hilário FRANCO JR. (Univ. de São Paulo)

- Leonardo fúnes (Univ. de Buenos Aires / SECRIT-CONICET)

- Ariel GUIANCE (CONICET)

- Patrick HENRIET (Univ. Bordeaux 3 ; SIREM)

- Alfonso hERNÁNDEZ (Univ. de Buenos Aires - CONICET)

- Dominique IOGNA-PRAT (CNRS, Auxerre/Dijon ; SIREM)

- Marta MADERo (Univ. Nac. de General Sarmiento, Argentine ; SIREM)

- Eliana MAGNANI (CNRS, Auxerre/Dijon)

- Georges MARTIN (Univ. Paris-Sorbonne ; SIREM)

- Pascual MARTínez SOPENA (Univ. de Valladolid)

- Paola miceli (Univ. de Buenos Aires -Univ. Nacional de General Sarmiento)

- Joseph MORSEL (Univ. Paris I)

- Márcia MONGELli (Univ. de São Paulo, Brésil)

- Sebastian PROVVIDENTE (Univ. de Buenos Aires -Univ. Nacional de General Sarmiento)

- José RIVAIR MACEDo (Univ. Federal do Rio Grande do Sul)

- Ana RODRíGUEZ LóPEZ (CSIC, Madrid)

- Gerardo RODRíGUEz (Univ. Nacional de Mar del Plata - Univ. Nacional del Sur, Argentine)

- Luis Rojas donat (Univ. de Bio-Bio, Chili) 
- Marcos RUBiolo galíndez (Universidad de Córdoba, Argentina)

- Daniel Russo (Univ. de Bourgogne)

- Jean-Claude schmitt (EHESS, Paris)

\section{Avec le soutien de :}

- CEM - Centre d'études médiévales d'Auxerre

- UMR 5594 -Archéologie, cultures et sociétés (Dijon - CNRS, Université de Bourgogne, Ministère de la Culture)

- Casa de Velázquez

- SIREM (Séminaire interdisciplinaire sur l'Espagne médiévale, GDR 2378)

- Institut Universitaire de France

- CNRS-Bureau pour le Cône Sud et pour le Brésil

- Agencia de Promoción a Investigación (Argentine)

- CONICET (Argentine)

- Departamento de Investigaciones Medievales, CONICET (Argentine)

NOTES

1. Ministère de la Recherche, Casa de Velázquez, École de hautes études en sciences sociales, Agence Universitaire de la francophonie, Institut Universitaire de France, Universidad de Buenos Aires, Universidade de São Paulo (Instituto de Estudos Avançados), Fundação de Amparo à Pesquisa do Estado de São Paulo (FAPESP). 